\section{Prevalência de sobrepeso e obesidade e fatores associados em escolares de área urbana de Chilpancingo, Guerrero, México, 2004}

\author{
Prevalence of overweight and obesity, and \\ associated factors in school children from urban \\ area in Chilpancingo, Guerrero, Mexico, 2004
}

\author{
Suzana Alves de Moraes 1 \\ Juana Beltrán Rosas 2 \\ Lenise Mondini 1,3 \\ Isabel Cristina Martins de Freitas 1
}

\footnotetext{
1 Escola de Enfermagem de Ribeirão Preto,

Universidade de São Paulo, Ribeirão Preto, Brasil.

2 Unidad Académica de

Enfermería no 1, Universidad Autónoma de Guerrero,

Chilpancingo, México.

3 Instituto de Saúde

Secretaria de Estado da Saúde, São Paulo, Brasil.

Correspondência

S. A. Moraes

Núcleo de Epidemiologia

Escola de Enfermagem

de Ribeirão Preto,

Universidade de São Paulo.

Av. dos Bandeirantes 3900 ,

São Paulo, SP

14040-902, Brasil.

samoraes@usp.br
}

\begin{abstract}
This study aimed to investigate the prevalence rates for overweight and obesity among Mexican schoolchildren, identifying factors associated with the outcomes. A cross-sectional study was conducted in a sample consisting of 700 schoolchildren. Prevalence rates were calculated according to age and gender, and odds ratios were estimated by point and intervals, using multivariate logistic regression. Overweight and obesity prevalence were $28.1 \%$ and $13.7 \%$, respectively. Risk factors for overweight were: mother's schooling (in years); eating while studying or watching $T V$; scores for risk food items $\geq 12$ points; sedentary activities $\geq 1.6$ hours/day, and birth weight $\geq 2,890$ grams. Obesity risk factors were similar to those related to overweight. Higher frequency of sport activities was detected as a protective factor in both outcomes. The associated factors for overweight and obesity highlighted the focus for health programs to prevent chronic diseases in this population, for which risk factors can already be identified in childhood.
\end{abstract}

Obesity; Nutricional Status; Risk Factors; Crosssectional Studies

\section{Introdução}

Diferentes autores têm estudado a transição nutricional na América Latina, procurando identificar sua relação com a Transição Demográfico-Epidemiológica (TDE). Uauy et al. 1, ao comparar países da América Latina como a Guatemala, o México, o Chile e o Uruguai constataram que, enquanto a Guatemala apenas recentemente iniciou o processo de transição epidemiológica, o México já se encontra no meio do processo, classificando-se em um estágio intermediário da TDE. O Chile finalizou, recentemente, o período conhecido como variante transicional rápida, identificado por Omran 2 como um dos modelos da TDE que caracterizam alguns países da América Latina. O Uruguai classifica-se entre aqueles cuja transição segue o modelo acelerado (semi-ocidental), e equivalente, na visão desses autores, ao período de pós-transição. Coincidindo com o padrão de transição epidemiológica, coexistem padrões específicos de transição nutricional: em países onde predominam os óbitos por doenças cardiovasculares a prevalência de obesidade é alta, enquanto em países com elevadas taxas de desnutrição predominam os óbitos por doenças infecciosas 1,2. A obesidade é decorrente da interação entre fatores genéticos e ambientais. Entre os fatores ambientais modificáveis e que contribuem para o balanço positivo de energia destacam-se a ingestão de ali- 
mentos de elevada densidade energética e a inatividade física 3 .

Em recente publicação da Organização PanAmericana da Saúde/Organização Mundial da Saúde ${ }^{4}$ para a América Latina, os autores reconheceram que a tendência de aumento da obesidade em áreas urbanas de países pobres, devia-se, principalmente, ao incremento da renda, em contraste com os países situados em níveis mais avançados de desenvolvimento, onde o aumento da renda resultou em tendência de declínio da obesidade.

No México, as estimativas sobre a obesidade infantil foram obtidas com base em dois estudos nacionais. $\mathrm{O}$ primeiro foi realizado no final da década de 80 , em crianças menores de cinco anos, residentes, predominantemente, em áreas rurais do país 5 . O segundo estudo, o National Nutrition Survey - 1999 6, teve como objetivos delinear o perfil da prevalência do sobrepeso e da obesidade em crianças e adultos do país, e identificar os fatores de risco mais importantes para o excesso de peso. Entre 1988 e 1999, o percentual de incremento da prevalência de sobrepeso, em menores de cinco anos, foi de $26,2 \%$, passando de $4,2 \%$ para $5,3 \%$, enquanto que na população adulta, do sexo feminino, os incrementos percentuais de sobrepeso e de obesidade foram, respectivamente, $46,7 \%$ (de $24 \%$ para $35,2 \%$ ) e $159 \%$ (de $9,4 \%$ para $24,4 \%$ ).

Hernandez et al. 7 , ao apresentarem os resultados do segundo estudo multicêntrico 6, para os escolares de 5 a 11 anos de quatro macrorregiões do México, relataram que as prevalências de excesso de peso (sobrepeso + obesidade) para as regiões urbana e rural foram, respectivamente, $22,9 \%$ e $11,7 \%$.

Na população adulta, a obesidade aumenta o risco de adoecer e morrer e, nas crianças vem se convertendo em um problema que terá profundas repercussões em Saúde Pública. A obesidade infantil é um tema que, recentemente, vem ganhando destaque, tanto em países desenvolvidos como em desenvolvimento, pois as crianças que apresentam sobrepeso poderão tornar-se adultos com excesso de peso 8 .

Embora as doenças associadas à obesidade ocorram mais freqüentemente em adultos, $o$ excesso de peso tem conseqüências relevantes, já na infância, destacando-se, entre outras, a hiperlipidemia, intolerância à glicose, esteatose hepática, hipertensão arterial, apnéia do sono, e complicações ortopédicas, entre outras 9 .

O presente estudo teve por objetivo verificar a prevalência de sobrepeso e obesidade em escolares de áreas urbanas residentes em Chilpancingo, México, em 2004, e identificar seus fatores de risco ou de proteção. Espera-se que os resultados possam contribuir para a delimitação do problema em área urbana, podendo revelar contribuições específicas de determinadas exposições para o excesso de peso.

\section{Material e métodos}

O estudo teve um delineamento transversal e a amostra foi composta por 700 escolares de ambos os sexos, matriculados em escolas primárias, localizadas na área urbana da Cidade de Chilpancingo, capital do estado de Guerrero, México. As escolas primárias correspondem ao primeiro ciclo de escolaridade formal, constituído por seis séries escolares, e o ingresso na primeira série ocorre aos seis anos de idade. Utilizou-se a técnica de amostragem probabilística, com partilha proporcional 10 em três estágios: (i) sorteio das escolas; (ii) sorteio das séries, segundo turnos escolares e (iii) sorteio dos escolares. O cálculo do tamanho da amostra foi efetuado considerandose prevalência de "excesso de peso" de $25 \%$ e uma margem de erro de $3 \%$. De um total de 59 escolas, sete foram sorteadas e a coleta de dados foi realizada no período de março a maio de 2004.

Para a detecção do sobrepeso e da obesidade (variáveis dependentes), utilizou-se o Índice de Massa Corporal (IMC; $\mathrm{kg} / \mathrm{m}^{2}$ ), e a classificação do estado nutricional, nas crianças e adolescentes, foi baseada nos pontos de corte dos valores de IMC, segundo sexo e idade, recomendados por Cole et al. 11 .

Para a aferição do peso, utilizaram-se balanças digitais da marca Tanita, modelo 1582, com capacidade para $120 \mathrm{~kg}$. A aferição da altura foi obtida por meio de estadiômetros de parede, marca Seca, modelo 208. Para a aferição das medidas antropométricas, aplicaram-se as técnicas recomendadas por Kaufer 12. As medidas foram tomadas por entrevistadores treinados e o controle de qualidade foi avaliado usando-se o método proposto por Habicht 13.

As variáveis de exposição foram agrupadas em quatro blocos:

- Variáveis sócio-demográficas: sexo (foram considerados ambos os sexos); idade em anos completos obtidos por meio do cálculo: [(data da entrevista - data de nascimento)/365,25]; escolaridade dos pais (em anos completos de escolaridade formal); e renda familiar per capita em dólares americanos (somatória da renda individual de cada membro da família/número total de integrantes da família). Para o cálculo das prevalências de sobrepeso e obesidade, a variável idade foi classificada em duas categorias: crianças ( $\leq 10$ anos) e adolescentes (> 10 anos) ${ }^{4}$. Na fase analítica (modelagem) a variável idade foi tratada sob a forma contínua. 
- Variáveis relacionadas ao padrão alimentar: número de refeições/dia; hábito de consumir as refeições em horários regulares; hábito de levar alimentos para a escola; hábito de comer na cantina da escola; hábito de comer estudando ou assistindo à TV foram classificadas sob a forma dicotômica. Os escores de alimentos de risco; consumo per capita de açúcar; consumo per capita de gordura de porco; consumo per capita de óleos e gasto, per capita, com alimentação em dólares americanos foram classificados em três categorias. Para as cinco últimas variáveis, com exceção do consumo de gordura de porco, as categorias foram definidas segundo os pontos de corte dos respectivos tercis. $\mathrm{O}$ hábito de consumir gordura de porco foi classificado em três categorias: "não consumidores", "consumidores de quantidades < 150g" e "consumidores de quantidades $\geq 150 \mathrm{~g}$ ". As categorias de exposição foram definidas segundo a mediana de consumo.

O inquérito de freqüência de consumo alimentar dos escolares, aplicado às mães, foi composto por alimentos extraídos da Lista do Sistema de Equivalentes 14, considerados como os mais utilizados pela população mexicana, e que constam da Norma Oficial Mexicana de 1999 15. Esses alimentos estão classificados em categorias de risco para doenças crônicas, sendo que os alimentos ou preparações culinárias com alta densidade energética e/ou de reduzido valor nutritivo foram classificados como de "risco" para o excesso de peso. No presente estudo, para cada alimento classificado como de "risco" foi calculado um "peso" de acordo com a sua freqüência de consumo. Com este cálculo obteve-se a freqüência diária de consumo de alimentos de "risco" em cada participante do estudo. A somatória de todos os "pesos" deu origem aos escores de freqüência de consumo de alimentos 16 .

- Atividade física: o hábito de praticar atividade esportiva fora da escola foi classificado sob a forma dicotômica (sim/não) e a freqüência do hábito foi definida como o número de vezes por semana em que os participantes praticavam atividade esportiva fora da escola. $\mathrm{O}$ hábito de caminhar para ir à escola foi classificado sob a forma dicotômica (sim/não). O tempo gasto em atividades sedentárias (número de horas por dia) foi classificado em três categorias, utilizando-se como pontos de corte os tercis de tempo.

O tempo gasto em atividades sedentárias, tais como: assistir à TV, assistir a filmes, brincar com videogames e usar o computador foi classificado segundo a freqüência (número de vezes por semana) e o tempo gasto durante cada sessão. A somatória do produto [(número de vezes/semana x tempo por sessão)/7] gerou a variável "número médio de horas dedicadas a atividades sedentárias/dia”, sendo esta variável classificada em tercis.

- Estado nutricional dos pais e peso ao nascer dos escolares: o estado nutricional dos pais foi classificado em três categorias: "normais", "com sobrepeso" e "obesos", utilizando-se os pontos de corte recomendados pela OMS para os valores de IMC na população adulta 17 . Informações sobre o peso ao nascer dos escolares foram extraídas de suas carteiras de vacinação, apresentadas pelas mães ou responsáveis no momento da entrevista. Esta variável foi classificada em três categorias utilizando-se como pontos de corte os tercis de peso.

Os dados coletados foram transferidos para um banco de dados previamente formatado no programa EPIDATA para Windows, versão 2.1b (Epidata Association, Odense, Dinamarca), por meio de dupla entrada. O gerenciamento e a análise dos dados foram efetuados no programa STATA versão 7.0 (Stata Corporation, College Station, Estados Unidos). Além das prevalências de sobrepeso e obesidade, segundo o sexo e a idade, foram também estimadas as prevalências de seus potenciais fatores de risco, nas três categorias do estado nutricional: "normais" "com sobrepeso" e "obesos".

As medidas de associação utilizadas foram os odds ratios, estimados por pontos e por intervalos com $95 \%$ de confiança. A técnica estatística utilizada para a obtenção das medidas de associação foi a regressão logística multivariada, desenvolvida em modelos uni e multivariados. Nos modelos univariados, as variáveis que apresentaram valores $\mathrm{p} \leq 0,25$ nos testes de Wald foram incluídas nos modelos subseqüentes 18 .

No processo de modelagem, optou-se por seguir uma seqüência de etapas, de acordo com as recomendações de Hosmer \& Lemeshow 18 para técnicas de regressão logística multivariada. Seguindo tais recomendações, as análises foram desenvolvidas em três etapas:

- Primeira etapa: os modelos foram constituídos pelas análises univariadas, contendo cada uma das variáveis preditoras e as variáveis resposta-desfecho. Nesta etapa, todas as variáveis cujos coeficientes de regressão $(\beta)$ ou seus respectivos odds ratios apresentaram valores p para os testes de Wald $\leq 0,25$ foram consideradas potenciais candidatas aos modelos subseqüentes; - Segunda etapa: os modelos subseqüentes foram desenvolvidos baseando-se em subconjuntos específicos e separados de variáveis, por exemplo: (i) modelos contendo apenas as variáveis sócio-demográficas e a variável respos- 
ta; (ii) modelos contendo apenas as variáveis relacionadas ao padrão alimentar e a variável resposta, e assim sucessivamente. As variáveis que nestes modelos, conhecidos como modelos de ajustamento parcial, apresentaram valores $\mathrm{p} \leq 0,10$ para os testes de Wald foram incluídas em modelos subseqüentes;

- Terceira etapa: foram construídos os modelos finais, nos quais permaneceram apenas as variáveis que apresentaram valores p para os testes de Wald $<0,05$.

As variáveis independentes, classificadas com mais de duas categorias, foram tratadas sob a forma "dummy" (variáveis indicadoras) o que permite o cálculo direto das medidas de associação (odds ratios) em estratos específicos, além da identificação de gradiente linear nos respectivos estratos. Durante todas as fases de desenvolvimento dos modelos multivariados, a relação entre o número de eventosresposta e o número de variáveis independentes foi mantida como $\geq 10: 119$.

O projeto de pesquisa foi submetido e aprovado pelo Comitê de Investigação da Universidad Autónoma de Guerrero. As entrevistas foram aplicadas após a assinatura, pelos responsáveis, do termo de consentimento informado.

\section{Resultados}

De um total de 700 escolares, 18 foram excluídos por apresentarem baixo peso (IMC $<$ percentil 5) 20 e 20 foram excluídos por seguirem algum tipo de dieta alimentar para perda de peso. A amostra do estudo foi constituída por 662 escolares, classificados em dois grandes grupos etários: crianças e adolescentes. No grupo de crianças, a média de idade foi de 8,06 anos, com valores mínimo e máximo, respectivamente de 5,69 e 9,99 anos. No grupo de adolescentes, a média de idade foi de 11,35 anos, sendo os valores mínimo e máximo de 10,01 e 13,22, respectivamente.

Na Tabela 1 apresenta-se a prevalência do estado nutricional dos escolares, segundo sexo e grupo etário. Entre as crianças, as prevalências de sobrepeso e obesidade no sexo masculino, foram, respectivamente, $20,6 \%$ e $17,1 \%$ e, para o sexo feminino $30,8 \%$ e $15,4 \%$. Entre os adolescentes, as prevalências de sobrepeso e obesidade no sexo masculino, foram, respectivamente, $26,6 \%$ e $14,4 \%$ e, para o sexo feminino $38,4 \%$ e $7,2 \%$. A prevalência de sobrepeso em crianças do sexo feminino foi maior que no masculino, o mesmo ocorrendo entre os adolescentes e, estas diferenças foram estatisticamente significantes $(p<0,05)$. Em relação à obesidade, as respectivas diferenças não apresentaram significância estatística.

Na Tabela 2 apresentam-se a prevalência (variáveis categóricas) e as médias (variáveis contínuas) dos potenciais fatores de risco/proteção para o sobrepeso e a obesidade. Do total de escolares classificados como obesos, 52,7\% pertenciam ao terceiro tercil de renda familiar per capita (renda $\geq$ US\$708,78), enquanto que apenas $24,2 \%$ dos classificados como normais encontravam-se na mesma condição.

Entre os obesos, observa-se que $81,3 \%$ comiam ou estudavam vendo TV, enquanto que cerca de 35,8\% dos normais adotavam este hábito; $61,5 \%$ dos escolares obesos pertenciam à categoria correspondente aos valores mais elevados dos escores de freqüência de consumo de alimentos de "risco"; 53,8\% dos escolares obesos viviam em domicílios com os mais altos gastos, per capita, com alimentação e 51,6\%

Tabela 1

Prevalência (\%) de sobrepeso e obesidade* em escolares, segundo sexo e faixa etária.

Chilpancingo, Guerrero, México, 2004.

\begin{tabular}{|c|c|c|c|c|c|c|c|c|}
\hline \multirow[t]{4}{*}{ Estado nutricional } & \multicolumn{8}{|c|}{ Faixa etária } \\
\hline & \multicolumn{4}{|c|}{ Crianças ( $\leq 10$ anos) } & \multicolumn{4}{|c|}{ Adolescentes (> 10 anos) } \\
\hline & \multicolumn{2}{|c|}{ Masculino } & \multicolumn{2}{|c|}{ Feminino } & \multicolumn{2}{|c|}{ Masculino } & \multicolumn{2}{|c|}{ Feminino } \\
\hline & $\mathrm{N}$ & $\%$ & $\mathrm{~N}$ & $\%$ & $\mathrm{~N}$ & $\%$ & $\mathrm{~N}$ & $\%$ \\
\hline Normal & 127 & 62,3 & 108 & 53,8 & 82 & 59,0 & 75 & 54,4 \\
\hline Sobrepeso & 42 & 20,6 & 62 & 30,8 & 37 & 26,6 & 53 & 38,4 \\
\hline Obesidade & 35 & 17,1 & 31 & 15,4 & 20 & 14,4 & 10 & 7,2 \\
\hline Total & 204 & 100,0 & 201 & 100,0 & 139 & 100,0 & 138 & 100,0 \\
\hline
\end{tabular}

* Os critérios utilizados para a classificação do sobrepeso e da obesidade em crianças e adolescentes seguem as recomendações de Cole et al. 11, que estabelecem pontos de corte específicos por sexo e idade para o IMC, equivalentes aos respectivos valores na população adulta. 
Prevalência (\%) e valores médios dos potenciais fatores de risco para o excesso de peso em escolares, segundo categorias do estado nutricional*. Chilpancingo, Guerrero, México, 2004

\begin{tabular}{|c|c|c|c|c|c|c|}
\hline \multirow[t]{2}{*}{ Variáveis } & \multicolumn{2}{|c|}{$\begin{array}{l}\text { Normal } \\
(n=385)\end{array}$} & \multicolumn{2}{|c|}{$\begin{array}{l}\text { Sobrepeso } \\
(n=186)\end{array}$} & \multicolumn{2}{|c|}{$\begin{array}{l}\text { Obesidade } \\
(n=91)\end{array}$} \\
\hline & $\%$ & Média $\pm d p^{\star \star}$ & $\%$ & Média $\pm d p^{\star \star}$ & $\%$ & Média $\pm d p^{\star \star}$ \\
\hline \multicolumn{7}{|l|}{ Sexo } \\
\hline Masculino & 52,7 & & 40,9 & & 57,1 & \\
\hline Feminino & 47,3 & & 59,1 & & 42,9 & \\
\hline Idade (em anos) & & $9,3 \pm 2,0$ & & $9,7 \pm 1,8$ & & $9,0 \pm 1,6$ \\
\hline $\begin{array}{l}\text { Escolaridade da mãe } \\
\text { (em anos completos) }\end{array}$ & & $10,3 \pm 4,1$ & & $11,8 \pm 4,2$ & & $11,9 \pm 3,5$ \\
\hline $\begin{array}{l}\text { Escolaridade do pai } \\
\text { (em anos completos) }\end{array}$ & & $11,6 \pm 4,2$ & & $13,2 \pm 4,2$ & & $13,5 \pm 3,9$ \\
\hline \multicolumn{7}{|c|}{ Renda familiar per capita (em US\$) } \\
\hline 1ㅇ tercil $(<442,99)$ & 41,0 & & 24,7 & & 16,5 & \\
\hline 2o tercil $(442,99+708,78)$ & 34,8 & & 34,4 & & 30,8 & \\
\hline 3o tercil $(\geq 708,78)$ & 24,2 & & 40,9 & & 52,7 & \\
\hline \multicolumn{7}{|l|}{ Número de refeições/dia } \\
\hline 3 a 5 & 93,0 & & 96,8 & & 96,7 & \\
\hline$<3$ & 7,0 & & 3,2 & & 3,3 & \\
\hline \multicolumn{7}{|l|}{ Horário regular para refeições } \\
\hline Sim & 65,2 & & 61,8 & & 58,2 & \\
\hline Não & 38,8 & & 38,2 & & 41,8 & \\
\hline \multicolumn{7}{|c|}{ Hábito de levar alimentos para a escola } \\
\hline Não & 69,6 & & 67,2 & & 49,4 & \\
\hline Sim & 30,4 & & 32,8 & & 50,6 & \\
\hline \multicolumn{7}{|c|}{ Hábito de comer na cantina da escola } \\
\hline Não & 26,0 & & 23,0 & & 31,9 & \\
\hline Sim & 74,0 & & 77,0 & & 68,1 & \\
\hline \multicolumn{7}{|c|}{$\begin{array}{l}\text { Hábito de comer enquanto estuda } \\
\text { ou assiste à TV }\end{array}$} \\
\hline Não & 64,2 & & 24,2 & & 18,7 & \\
\hline Sim & 35,8 & & 75,8 & & 81,3 & \\
\hline \multicolumn{7}{|c|}{$\begin{array}{l}\text { Escores de freqüência de alimentos } \\
\text { de "risco"\# }\end{array}$} \\
\hline 1o tercil $(<12,0)$ & 50,4 & & 10,7 & & 5,5 & \\
\hline 2o tercil $(12,0 \vdash 15,0)$ & 33,5 & & 33,9 & & 33,0 & \\
\hline 3o tercil $(\geq 15,0)$ & 16,1 & & 55,4 & & 61,5 & \\
\hline \multicolumn{7}{|l|}{$\begin{array}{l}\text { Consumo domiciliar de açúcar } \\
\text { (g/per capita/mês) }\end{array}$} \\
\hline 1 으 tercil $(<416)$ & 40,0 & & 26,9 & & 17,6 & \\
\hline 2 o tercil $(416+583)$ & 29,1 & & 34,4 & & 30,8 & \\
\hline 3o tercil $(\geq 583)$ & 30,9 & & 38,7 & & 51,6 & \\
\hline \multicolumn{7}{|l|}{$\begin{array}{l}\text { Consumo domiciliar de gordura } \\
\text { de porco (g/per capita/mês) }\end{array}$} \\
\hline Não consome & 72,5 & & 53,8 & & 51,6 & \\
\hline$<150 \mathrm{~g}$ & 19,2 & & 23,6 & & 15,4 & \\
\hline$\geq 150 \mathrm{~g}$ & 8,3 & & 22,6 & & 33,0 & \\
\hline \multicolumn{7}{|l|}{$\begin{array}{l}\text { Consumo domiciliar de óleo } \\
\text { (ml/per capita/mês) }\end{array}$} \\
\hline 1ㅇ tercil $(<375)$ & 38,5 & & 28,5 & & 24,1 & \\
\hline 2o tercil $(375+500)$ & 41,0 & & 42,5 & & 40,7 & \\
\hline 3o tercil $(\geq 500)$ & 20,5 & & 29,0 & & 35,2 & \\
\hline
\end{tabular}

(continua) 


\begin{tabular}{|c|c|c|c|c|c|c|}
\hline \multirow[t]{2}{*}{ Variáveis } & \multicolumn{2}{|c|}{$\begin{array}{l}\text { Normal } \\
(n=385)\end{array}$} & \multicolumn{2}{|c|}{$\begin{array}{l}\text { Sobrepeso } \\
(n=186)\end{array}$} & \multicolumn{2}{|c|}{$\begin{array}{l}\text { Obesidade } \\
(\mathrm{n}=91)\end{array}$} \\
\hline & $\%$ & Média $\pm d p^{\star \star}$ & $\%$ & Média $\pm d p^{\star \star}$ & $\%$ & Média $\pm d p^{* *}$ \\
\hline \multicolumn{7}{|l|}{$\begin{array}{l}\text { Gasto com alimentação no último } \\
\text { mês (per capita em US\$) }\end{array}$} \\
\hline 1으 tercil $(<33,22)$ & 42,1 & & 23,1 & & 19,8 & \\
\hline 2o tercil $(33,22+44,30)$ & 32,5 & & 33,3 & & 26,4 & \\
\hline 3o tercil $(\geq 44,30)$ & 25,4 & & 43,6 & & 53,8 & \\
\hline \multicolumn{7}{|l|}{ Atividade esportiva fora da escola } \\
\hline $\operatorname{Sim}$ & 57,1 & & 40,3 & & 44,0 & \\
\hline Não & 42,9 & & 59,7 & & 56,0 & \\
\hline $\begin{array}{l}\text { Atividade esportiva fora da } \\
\text { escola (número de vezes/semana) }\end{array}$ & & $1,93 \pm 1,9$ & & $0,94 \pm 1,3$ & & $0,91 \pm 1,3$ \\
\hline \multicolumn{7}{|l|}{ Hábito de ir à escola caminhando } \\
\hline $\operatorname{Sim}$ & 63,6 & & 45,7 & & 35,2 & \\
\hline Não & 36,4 & & 54,3 & & 64,8 & \\
\hline \multicolumn{7}{|c|}{ Atividades sedentárias (em horas/dia) } \\
\hline 1ㅇ tercil $(<1,6)$ & 51,4 & & 10,2 & & 4,4 & \\
\hline 2o tercil $(1,6 \vdash 2,9)$ & 35,6 & & 33,3 & & 31,9 & \\
\hline 3으 tercil $(\geq 2,9)$ & 13,0 & & 56,5 & & 63,7 & \\
\hline \multicolumn{7}{|l|}{ Peso ao nascer (em gramas) } \\
\hline 1ㅇ tercil $(2.501 \vdash 2.890)$ & 47,0 & & 10,2 & & 7,7 & \\
\hline 2o tercil $(2.890 \vdash 3.110)$ & 39,5 & & 33,3 & & 19,8 & \\
\hline 3o tercil ( $\geq 3.110$ ) & 13,5 & & 56,5 & & 72,5 & \\
\hline \multicolumn{7}{|l|}{ Estado nutricional da mãe } \\
\hline Normal & 64,7 & & 50,0 & & 52,7 & \\
\hline Sobrepeso & 33,5 & & 47,8 & & 40,7 & \\
\hline Obesidade & 1,8 & & 2,2 & & 6,6 & \\
\hline \multicolumn{7}{|l|}{ Estado nutricional do pai } \\
\hline Normal & 57,5 & & 41,8 & & 28,6 & \\
\hline Sobrepeso & 40,3 & & 53,1 & & 58,3 & \\
\hline Obesidade & 2,2 & & 5,1 & & 13,1 & \\
\hline
\end{tabular}

* Os critérios utilizados para a classificação do sobrepeso e da obesidade em crianças e adolescentes seguem as recomendações de Cole et al. 11, que estabelecem pontos de corte específicos por sexo e idade para o IMC, equivalentes aos respectivos valores na população adulta; ** $\mathrm{dp}=$ desvio padrão;

*** Conversão de pesos mexicanos em dólares americanos (1 US $\$=11,2870$ pesos mexicanos):

\# Alimentos e preparações culinárias de alta densidade energética e alimentos de reduzido valor nutritivo.

naqueles com maior consumo de açúcar. Com relação às variáveis relacionadas à atividade física, observa-se que $54,3 \%$ e $64,8 \%$ dos escolares com sobrepeso e obesidade, respectivamente, não costumavam caminhar para ir à escola; $56,5 \%$ daqueles com sobrepeso e $63,7 \%$ dos obesos encontravam-se na categoria correspondente aos valores mais elevados de tempo dedicado a atividades sedentárias ( $\geq 2,9$ horas/dia); os escolares com sobrepeso ou obesidade dedicavam-se à prática de atividades esportivas fora da escola, numa freqüência média menor que uma vez por semana, enquanto que entre os escolares classificados como normais, tal freqüência foi, aproximadamente, duas vezes por semana; $56,5 \%$ dos escolares com sobrepeso e $72,5 \%$ dos obesos nasceram com peso $\geq 3.110$ g (3o tercil de peso ao nascer);
$53,1 \%$ dos escolares com sobrepeso e $58,3 \%$ dos obesos eram filhos de pais com sobrepeso.

Na Figura 1 apresenta-se a reta de regressão para os escores de freqüência de consumo de alimentos de "risco", os coeficientes angulares $(\beta)$ e respectivos valores " $p$ ", segundo a idade dos escolares, nas três categorias do estado nutricional. Observa-se um aumento da freqüência de consumo de alimentos de "risco" à medida que aumenta a idade, mesmo entre aqueles classificados como "normais". Os coeficientes angulares $(\beta)$ apresentaram um gradiente linear $(0,23 ; 0,54$ e 0,65$)$ nas categorias "normal", "sobrepeso" e "obesidade", respectivamente, e, em cada uma dessas categorias foram estatisticamente significantes $(\mathrm{p}<0,05)$.

Antes do desenvolvimento do processo de modelagem, algumas interações de interesse 
foram testadas: "atividades sedentárias (em horas/dia)" versus "hábito de comer enquanto estuda ou assiste à TV" e "atividades sedentárias (em horas/dia)" versus "escores de freqüência de consumo de alimentos de risco". Considerando-se que tais interações não apresentaram significância estatística ( $p>0,05)$, prosseguiuse com o processo de modelagem para a avaliação do efeito de confusão.

Verifica-se na Tabela 3 que os fatores de risco para o sobrepeso compuseram o seguinte elenco: número de anos de escolaridade da mãe; hábito de comer enquanto estudavam ou assistiam à TV; escores de freqüência de alimentos de "risco" classificados no 2 o e 3 o tercis de consumo ( $\geq 12$ pontos); atividades sedentárias no 2 o e 3 o tercis ( $\geq 1,6$ horas/dia); e peso ao nascer no 2 o e 3 o tercis $(\geq 2.890 \mathrm{~g})$. A prática de atividades esportivas, fora da escola, foi identificada como fator de proteção.

Os fatores de risco para a obesidade (Tabela 4) compuseram o seguinte elenco: escores de freqüência de alimentos de "risco" classifica-

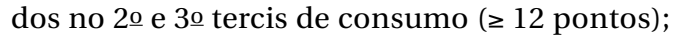
atividades sedentárias no 2 o e 3 o tercis $(\geq 1,6$ horas/dia); peso ao nascer no 3 o tercil $(\geq 3.110 \mathrm{~g})$; e a última categoria do estado nutricional do pai. A prática de atividades esportivas fora da escola foi também identificada como fator de proteção.

\section{Discussão}

A prevalência de sobrepeso e obesidade nos escolares do presente estudo foi maior que a encontrada por Hernandez et al. 7, no National Nutrition Survey de 1999, em crianças em idade escolar e residentes em áreas urbanas. Em decorrência da Transição Nutricional, os indicadores estimados em 2004 para Chilpancingo já ultrapassaram, em magnitude, os valores detectados para a prevalência de excesso de peso em áreas urbanas, encontrados no National Nutrition Survey de 1999. No presente estudo, a opção de incluir apenas crianças de áreas urbanas justifica-se por: (i) a Transição Nutricional vem ocorrendo de modo mais marcante nessas regiões em relação às áreas rurais; (ii) na pesquisa do National Nutrition Survey, embora a representatividade urbano/rural estivesse garantida no nível nacional, a desagregação destes dois níveis não pode ser garantida nos níveis estadual, municipal ou local e; (iii) na Cidade de Chilpancingo as escolas primárias concentram-se em áreas urbanas, sendo que a porção rural do município é pequena e quase inexistente.

\section{Figura 1}

Dispersão e reta de regressão dos escores de consumo de alimentos de "risco" para o excesso de peso, segundo a idade, nas três categorias do estado nutricional dos escolares. Chilpancingo, Guerrero, México, 2004.

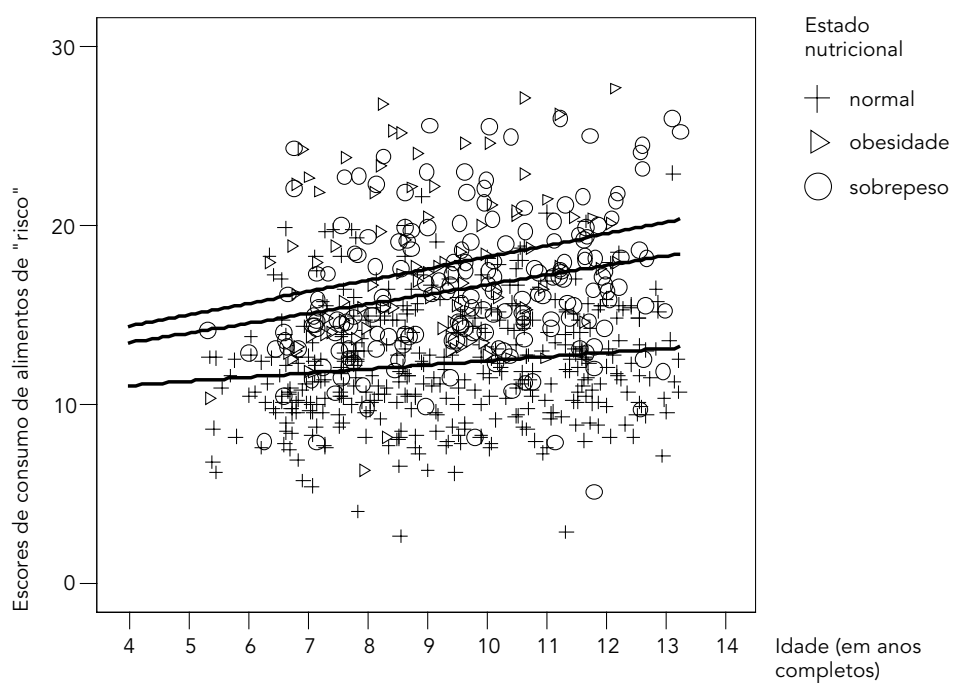

Normal: $\beta=0,23, p=0,000$; Obesidade: $\beta=0,65, p=0,03$; Sobrepeso: $\beta=0,54, p=0,00$.

Kain et al. 21, em um estudo de revisão sobre a tendência da obesidade e de seus fatores risco, reconheceram que a obesidade vem apresentando uma tendência de ascensão na América Latina, a partir da década de 90, e que o aumento da prevalência em escolares vem apresentando um deslocamento para as últimas séries do curso primário. Os autores reconhecem que os principais determinantes da obesidade são: condições fetais e de nutrição infantil; nível educacional; condições sócio-econômicas; mudanças no padrão dietético; e atividade sedentária.

Colleman 22, em estudo longitudinal conduzido em duas cidades localizadas em região de fronteiras (El Paso, Texas, Estados Unidos e Chihuahua, México), em 2001, investigou a prevalência de sobrepeso e obesidade em escolares de ambas as cidades, matriculados na 3ạ e 4a séries de escolas primárias. O autor identificou que em El Paso a prevalência de obesidade em meninos elevou-se de $22 \%$ para $28 \%$, e a de sobrepeso de $37 \%$ para $44 \%$, da 3 a para 4a série. Entre as meninas, a prevalência de obesidade elevou-se de $16 \%$ para $18 \%$, enquanto que o sobrepeso passou de $29 \%$ para $37 \%$, no mesmo período. Na Cidade de Chihuahua, o autor verificou que as respectivas prevalências, 
Fatores associados ao sobrepeso: odds ratios (OR) brutos e ajustados com respectivos intervalos de confiança (modelo final). Chilpancingo, Guerrero, México, 2004.

\begin{tabular}{|c|c|c|c|c|}
\hline Variáveis & OR bruto & IC95\% & OR ajustado & IC95\% \\
\hline Escolaridade da mãe (em anos completos) & 1,09 & $1,04-1,14$ & 1,07 & $1,01-1,14$ \\
\hline \multicolumn{5}{|c|}{ Hábito de comer enquanto estuda ou assiste à TV } \\
\hline Não & 1 & & 1 & \\
\hline Sim & 5,61 & $3,77-8,32$ & 2,41 & $1,45-4,01$ \\
\hline \multicolumn{5}{|l|}{ Escores de freqüência de alimentos de "risco"* } \\
\hline 1으 tercil $(<12,0)$ & 1 & & 1 & \\
\hline 2 o tercil $(12,0 \vdash 15,0)$ & 4,74 & $2,73-8,21$ & 3,82 & $2,02-7,20$ \\
\hline 3o tercil $(\geq 15,0)$ & 16,11 & $9,22-28,15$ & 4,84 & $2,47-9,50$ \\
\hline $\begin{array}{l}\text { Atividade esportiva fora da escola } \\
\text { (número de vezes por semana) }\end{array}$ & 0,71 & $0,64-0,80$ & 0,78 & $0,67-0,91$ \\
\hline \multicolumn{5}{|l|}{ Atividades sedentárias (em horas/dia) } \\
\hline 1으 tercil $(<1,6)$ & 1 & & 1 & \\
\hline 2o tercil $(1,6 \vdash 2,9)$ & 4,72 & $2,70-8,24$ & 2,80 & $1,51-5,21$ \\
\hline 3o tercil $(\geq 2,9)$ & 21,88 & $12,27-39,04$ & 7,23 & $3,57-14,64$ \\
\hline \multicolumn{5}{|l|}{ Peso ao nascer (em gramas) } \\
\hline 1ㅇ tercil $(2.501+2.890)$ & 1 & & 1 & \\
\hline 2 o tercil $(2.890+3.110)$ & 3,88 & $2,22-6,78$ & 2,85 & $1,49-5,47$ \\
\hline 3o tercil $(\geq 3.110)$ & 19,24 & $10,79-34,28$ & 7,03 & $3,53-13,99$ \\
\hline
\end{tabular}

* Alimentos e preparações culinárias de alta densidade energética e alimentos de reduzido valor nutritivo.

segundo o sexo, apresentaram valores similares aos encontrados em El Paso.

Ariza et al. 23, em estudo transversal conduzido com 250 crianças americanas de origem mexicana, em idade pré-escolar (5-6 anos) e residentes em áreas urbanas de Chicago, em 1996, detectaram prevalência de sobrepeso da ordem de $23 \%$. Entre os fatores de risco incluídos no estudo, os autores identificaram que assistir à TV por mais que 3 horas/semana (OR = 3,7; IC95\%: 1,2-11,0) e consumir diariamente refrigerantes (OR = 5,9; IC95\%: 1,2-29) foram considerados importantes preditores para o sobrepeso. Embora vários estudos tenham atribuído à aculturação um importante papel no excesso de peso em populações migrantes, esta variável não apresentou associação estatisticamente significante com o sobrepeso, neste estudo.

Em um estudo de rastreamento de obesidade realizado nos Estados Unidos entre 2000 e 2001, envolvendo 385 escolares da $6 \underline{a}$ e $7 \underline{\text { a }}$ séries de diferentes etnias, os autores apontaram prevalência de excesso de peso (definido como IMC acima do percentil 85 ) de $35,3 \%$, sendo que metade destes $(17,4 \%)$ eram obesos (IMC acima do percentil 95). Ao compararem as médias do escore $\mathrm{z}$ de IMC entre os latinos, bran- cos não-hispânicos e os de origem asiática, os autores constataram que essas médias foram mais elevadas entre os latinos $(p<0,001)$ que assistiam, em média, 2,4 horas/noite de TV, enquanto que os brancos não-hispânicos e os de origem asiática apresentaram média de 1,3 hora/noite 24 .

Ribeiro et al. 25, em um estudo caso-controle desenvolvido na Cidade de São Paulo, Brasil, em 2000, ao investigarem os fatores de risco para a obesidade em 446 crianças de 7 a 10 anos, matriculadas em oito escolas públicas desse município, identificaram, em modelos multivariados, que os principais fatores de risco para a obesidade foi constituído pelo seguinte elenco: peso ao nascer $\geq 3.500 \mathrm{~g}(\mathrm{OR}=1,83)$; bom apetite nas refeições $(\mathrm{OR}=3,81)$; assistir à $\mathrm{TV}$ por quatro horas ou mais por dia $(\mathrm{OR}=2,07)$; 4 anos ou mais de escolaridade da mãe (OR = $1,85)$; e obesidade dos pais $(\mathrm{OR}=2,50)$. Estes achados corroboram os resultados encontrados no presente estudo, onde o elenco de variáveis elegíveis para os modelos finais foi muito semelhante.

Giugliano \& Carneiro 26 identificaram prevalência de excesso de peso da ordem de $21,1 \%$ para meninos e de $22,9 \%$ para as meninas, em 
Fatores associados à obesidade: odds ratios (OR) brutos e ajustados com respectivos intervalos de confiança (modelo final). Chilpancingo, Guerrero, México, 2004.

\begin{tabular}{|c|c|c|c|c|}
\hline Variáveis & OR bruto & IC95\% & OR ajustado & IC95\% \\
\hline \multicolumn{5}{|c|}{ Escores de freqüência de alimentos de "risco"* } \\
\hline 1ㅇ tercil $(<12,0)$ & 1 & & 1 & \\
\hline 2 o tercil $(12,0 \vdash 15,0)$ & 9,02 & $3,41-23,86$ & 5,32 & $1,80-5,76$ \\
\hline 3으 tercil $(\geq 15,0)$ & 35,05 & $13,44-91,39$ & 11,18 & $3,70-33,76$ \\
\hline $\begin{array}{l}\text { Atividade esportiva fora da escola } \\
\text { (número de vezes por semana) }\end{array}$ & 0,71 & $0,61-0,82$ & 0,76 & $0,61-0,94$ \\
\hline \multicolumn{5}{|l|}{ Atividades sedentárias (em horas/dia) } \\
\hline 1o tercil $(<1,6)$ & 1 & & 1 & \\
\hline 2o tercil $(1,6 \vdash 2,9)$ & 10,48 & $3,60-30,48$ & 6,06 & $1,91-19,21$ \\
\hline 3o tercil $(\geq 2,9)$ & 57,42 & $19,90-165,67$ & 11,76 & $3,45-40,07$ \\
\hline \multicolumn{5}{|l|}{ Peso ao nascer (em gramas) } \\
\hline 1ㅇ tercil $(2.501 \vdash 2.890)$ & 1 & & 1 & \\
\hline 2 o tercil $(2.890+3.110)$ & 3,06 & $1,25-7,53$ & 1,79 & $0,62-5,18$ \\
\hline 3o tercil $(\geq 3.110$ ) & 32,82 & $14,20-75,86$ & 7,91 & $2,83-22,09$ \\
\hline \multicolumn{5}{|l|}{ Estado nutricional do pai } \\
\hline Normal & 1 & & 1 & \\
\hline Sobrepeso & 2,91 & $1,71-4,95$ & 2,02 & $0,98-4,13$ \\
\hline Obesidade & 11,92 & $4,37-32,52$ & 7,13 & $1,61-31,58$ \\
\hline
\end{tabular}

* Alimentos e preparações culinárias de alta densidade energética e alimentos de reduzido valor nutritivo.

452 escolares do Rio de Janeiro, Brasil, em 2003. A adiposidade estimada pela espessura da dobra cutânea tricipital e subescapular correlacionou-se diretamente com o tempo de permanência sentado e, inversamente, com o número de horas de sono $(p<0,05)$. A prevalência de sobrepeso e obesidade foi maior nos escolares cujas mães apresentavam baixa escolaridade ( $\mathrm{p}<0,01)$ e cujos pais apresentavam sobrepeso ou obesidade $(\mathrm{p}<0,01)$.

O'Loughlin et al. 27, em um estudo de coorte desenvolvido em uma população de 2.318 escolares entre 9 e 12 anos, em Montreal, Canadá, procurou identificar os principais preditores para os incrementos do IMC (variável resposta), em dois anos de observação. Após um ano de seguimento e em modelos multivariados, os autores identificaram nos meninos que o principal preditor foi o IMC acima do percentil 90, no início do seguimento $(\mathrm{OR}=2,66)$. Por outro lado, e no mesmo período, os principais preditores de incremento para as meninas, além do IMC acima do percentil $90(\mathrm{OR}=2,34)$ foram: não praticar esportes fora da escola $(\mathrm{OR}=$ $1,90)$ e jogar videogames diariamente $(\mathrm{OR}=$ $2,48)$. Ao final do segundo ano de seguimento, os OR para a condição "IMC acima do percentil
90" no início do seguimento foram, respectivamente, 3,26 e 2,22 em meninos e meninas, sendo que, para ambos os sexos as atividades sedentárias voltaram a ser identificadas como preditores independentes da variável resposta. Estes resultados sugerem que a obesidade apresentou um efeito cumulativo, ao longo do período de observação, em crianças de ambos os sexos, além de evidenciar que o sedentarismo exerceu seu efeito, já no primeiro ano para as meninas, enquanto que para os meninos tal efeito só pôde ser evidenciado ao final do segundo ano de seguimento.

Wake et al. 28, em um estudo transversal que arrolou 2.862 escolares entre 5 e 13 anos em Vitória, Austrália, relataram que o tempo dedicado ao hábito de assistir à TV, em horas/semana (OR $=1,45$ e 1,84, respectivamente, para 11-20h e $21-30 \mathrm{~h} /$ semana) constituiu-se em fator de risco independente para o excesso de peso (sobrepeso+obesidade), após o ajustamento para o IMC da mãe, escolaridade materna, número de irmãos, escores de freqüência de alimentos ingeridos/semana e atividade física.

Mo-suwan \& Geater 29 em um estudo transversal realizado na Tailândia, em 1996, estudaram 2.161 escolares de 6 a 13 anos completos, 
com o propósito de investigar fatores associados à obesidade infantil. Em modelos multivariados identificaram como fatores de risco independentes: história familiar de obesidade $(\mathrm{OR}=3,1)$; pouca atividade física $(\mathrm{OR}=2,6)$; mãe obesa $(\mathrm{OR}=1,9)$ e pai obeso $(\mathrm{OR}=2,2)$. Os autores calcularam também a fração de risco atribuível na população (RAP), identificando que a história familiar de obesidade apresentou RAP $=34 \%$; pouca atividade física, RAP = $12 \%$, e a condição de sobrepeso e obesidade da mãe, RAP $=10 \%$.

Stettler et al. 30 , em um estudo transversal onde foram incluídas 872 crianças, de 1a a $3 \underline{a}$ séries de escolas primárias, localizadas na parte alemã de Zurique, Suíça, em 1999, investigaram a associação de fatores ambientais e comportamentais associados com a obesidade. A obesidade foi definida a partir da combinação de dois indicadores (IMC e somatória das medidas de duas pregas cutâneas: escapular e a tricipital). Entre os fatores de risco/proteção detectados no estudo, os autores relataram: número de horas/dia em que utilizavam jogos eletrônicos (OR = 2,03; IC95\%: 1,57-2,61); número de horas/dia em que assistiam à $\mathrm{TV}(\mathrm{OR}=2,83$; IC95\%: 2,08-3,86); mãe que trabalha fora (OR = 1,93; IC95\%: 1,13-3,29); hábito de fumar do pai (OR = 1,78; IC95\%: 1,07-2,96) e escores de atividade física (OR = 0,80; IC95\%: 0,72-0,88).

Ekelund et al. 31, em um estudo transversal realizado em quatro regiões da Europa (Odense, Dinamarca; Ilha da Madeira, Portugal; Oslo, Noruega; e Tartu, Estônia), que incluiu 1.292 crianças entre 9 e 10 anos de idade, identificaram associação inversa entre o tempo gasto em atividade física e a quantidade de gordura corporal, após o ajustamento para sexo, maturidade sexual, peso ao nascer e o IMC dos pais $(\mathrm{p}<0,05)$.

Nos escolares de Chilpancingo, o número de horas/dia em atividades sedentárias (tercis de tempo) esteve associado com o sobrepeso e a obesidade ( $\mathrm{p}<0,05)$, após o ajustamento para importantes preditores do excesso de peso. Além disso, verificou-se um gradiente linear (efeito dose-resposta), expresso pelo aumento da magnitude dos OR, nas categorias de tempo dedicado às atividades sedentárias.

Os resultados das análises multivariadas, no presente estudo, indicaram que o peso ao nascer, a prática de atividade física, o número médio de horas diárias em atividades sedentárias e valores elevados para os escores de freqüência de consumo de alimentos de "risco" se constituíram em preditores independentes do sobrepeso e da obesidade em escolares de Chilpancingo, estando os resultados em consonância com aqueles encontrados na revisão da li- teratura. Torna-se importante mencionar que, além do "tempo gasto em atividades sedentárias", os odds ratios nas diferentes categorias das variáveis "escores de freqüência de consumo de alimentos de risco" e "peso ao nascer" apresentaram gradiente linear, o que reforça o seu papel na determinação dos desfechos aqui considerados.

Fornés et al. 16 propuseram a conversão de categorias de freqüência de alimentos, tradicionalmente utilizadas em estudos relacionados ao padrão alimentar, em escores de consumo, permitindo assim o tratamento da variável correspondente em uma escala contínua. Esta estratégia apresenta a vantagem de maximizar a utilização das informações relacionadas ao consumo alimentar, além de permitir a sua inclusão em modelos multivariados, desde que o espectro de variação dos escores apresente distribuição normal, o que pode ser identificado no presente estudo. Os escores calculados para o grupo de alimentos de risco para o excesso de peso refletem, de maneira sintética, a qualidade da dieta e o padrão de consumo desses alimentos, por meio da pontuação obtida. Em outras palavras, essa pontuação leva em consideração o tipo de alimento e sua respectiva freqüência, de forma simultânea, não havendo necessidade de classificar o consumo em categorias de freqüência tradicionalmente utilizadas em inquéritos alimentares.

Com relação ao estado nutricional dos pais, esta variável permaneceu nos modelos finais, apenas ao se considerar a obesidade como variável resposta e, nestes modelos, houve também indicação de um gradiente linear.

Os estudos com delineamento transversal são susceptíveis ao viés de causalidade reversa 32 , visto que a aferição das exposições e dos desfechos ocorre de modo simultâneo, ao contrário dos delineamentos clássicos em epidemiologia para a investigação de causalidade (estudos de coorte e estudos caso-controle). Entretanto, o encontro das associações aqui presentes, confirmam os achados dos estudos com objetivos similares e com delineamento do tipo caso-controle e coorte 25,27 , o que vem referendar a importância destes fatores para o desenvolvimento do excesso de peso em crianças e adolescentes. Um outro aspecto relevante que merece ser destacado é o fato de que, no presente estudo, além da detecção da prevalência de sobrepeso e obesidade nos escolares de Chilpancingo, procurou-se identificar os principais fatores de risco para esses eventos-resposta, utilizando-se modelos multivariados que, por seus pressupostos, permitem o ajustamento simultâneo para potenciais fatores de confusão. 
Torna-se também oportuno mencionar que durante a fase de planejamento do estudo procurou-se incluir, no instrumento de coleta dos dados, o elenco de preditores considerados de maior relevância para os eventos-resposta.

Atualmente, vários estudos têm sido publicados na literatura $33,34,35,36,37,38$ com o propósito de investigar o efeito do hábito de assistir à TV sobre a obesidade e o sobrepeso em crianças e adolescentes. Além de favorecer o hábito sedentário, tal comportamento poderia exercer seu efeito sobre o excesso de peso em virtude do apelo veiculado pela mídia em comerciais da indústria de alimentos, que podem favorecer o consumo de produtos ricos em gorduras hidrogenadas (gorduras "trans") pelas crianças 39 . No presente estudo, a variável "hábito de comer enquanto estuda ou assiste à TV" permaneceu no modelo final quando o evento resposta foi o sobrepeso.

A escolaridade da mãe, tratada, neste estudo, sob a forma contínua (número de anos de educação formal) esteve associada com o sobrepeso (OR = 1,07; IC95\%: 1,01-1,14), constituindo-se em bom preditor da variável resposta e indicando que a chance de sobrepeso nessas crianças aumentava 1,07 vezes para cada ano de escolaridade da mãe. É possível supor que as mães com maior escolaridade estejam inseridas no mercado de trabalho, o que poderia favorecer hábitos alimentares não muito saudáveis por parte dessas crianças. Achados semelhantes foram também relatados por Ribeiro et al. 25 e Stettler et al. 30 .

Os resultados do presente estudo não parecem ser resultantes de vícios de seleção, confusão ou aferição. O rigor metodológico aplicado ao processo de amostragem, ao treinamento dos entrevistadores para a aferição das medidas antropométricas e para a aplicação do questionário, bem como o desenvolvimento das etapas relacionadas ao gerenciamento e análise dos dados, conferiram validade interna ao estudo.

Os resultados da presente investigação permitiram a identificação dos principais fatores associados ao sobrepeso e à obesidade em escolares de zona urbana de Chilpancingo, indicando o direcionamento de programas de intervenção sobre esses desfechos e que possam contribuir para a prevenção de doenças crônicas nessa população, cujos fatores de risco já são identificados a partir da infância.

\section{Resumo}

Investigou-se a prevalência de sobrepeso e obesidade e respectivos fatores associados em escolares mexicanos. $O$ estudo teve delineamento transversal e amostra composta por 700 escolares. As prevalências de sobrepeso e obesidade foram calculadas segundo sexo e idade, e as medidas de associação (odds ratios) estimadas por pontos e por intervalos, utilizando-se a regressão logística multivariada. Para o conjunto de escolares, as prevalências de sobrepeso e obesidade foram $28,1 \%$ e 13,7\%, respectivamente. Os fatores de risco para o sobrepeso foram: "número de anos de escolaridade da mãe"; "hábito de comer enquanto estuda ou assiste à $T V$ "; "escores de freqüencia de consumo de alimentos de risco $\geq 12$ pontos"; "atividades sedentárias $\geq 1,6$ horas/dia" e "peso ao nascer $\geq 2.890$ gramas". Os fatores de risco para a obesidade foram semelhantes àqueles detectados para o sobrepeso. Maior freqüência de atividades esportivas foi identificada como fator de proteção para ambos os desfechos. A detecção dos principais fatores associados ao sobrepeso e à obesidade indica o direcionamento de intervenções para a prevenção de doenças crônicas nessa população, cujos fatores de risco já podem ser identificados a partir da infância.

Obesidade; Estado Nutricional; Fatores de Risco; Estudos Transversais 


\section{Colaboradores}

S. A. Moraes e I. C. M. Freitas participaram do planejamento do estudo e da elaboração do projeto de pesquisa, desenvolveram e aplicaram "rotinas" para o controle de qualidade das informações, coordenaram as etapas de gerenciamento dos dados, efetuaram as análises estatísticas e participaram da redação de todas as versões do manuscrito. J. B. Rosas participou do planejamento do estudo e da elaboração do projeto de pesquisa, supervisionou o trabalho de campo para a coleta de dados, acompanhou o desenvolvimento das análises estatísticas e colaborou na redação do manuscrito. L. Mondini participou do planejamento do estudo e da elaboração do projeto de pesquisa, colaborou nas análises estatísticas e na redação de todas as versões do manuscrito.

\section{Referências}

1. Uauy R, Albala C, Kain J. Obesity trends in Latin America: transiting under-to overweight. J Nutr 2001; 131:893S-9S.

2. Omran AR. The epidemiologic transition in the Americas. Washington DC: Pan-American Health Organization/The University of Maryland at College Park; 1996.

3. Pi-Sunyer FX. Obesity. In: Shills ME, Olson JA, Shike M, editors. Modern nutrition in health and disease. $8^{\text {th }}$ Ed. Philadelphia: Lea \& Febiger; 1994. p. 984-1001.

4. Pan-American Health Organization/World Health Organization. Obesity and poverty: a new public health challenge. Washington DC: Pan American Health Organization; 2000. (PAHO Scientific Publication, 576).

5. Rivera JA, Sepúlveda J. Conclusiones de la Encuesta Nacional de Nutrición 1999: traduciendo resultados en políticas públicas sobre nutrición. Salud Publica Méx 2003; 45 Suppl 4:S565-75.

6. Barquera S, Rivera JA, Safdie M, Flores M, Campos-Norato I, Campirano F. Energy and nutrient intake in preschool and school age Mexican children: National Nutrition Survey, 1999. Salud Pública Méx 2003; 45 Suppl 4:S540-50.

7. Hernández B, Cuevas-Nasu L, Shamah-Levy T, Monterrubio E, Ramírez-Silva C, García-Feregrino R, et al. Factors associated with overweight and obesity in Mexican school - age children: results from the National Nutrition Survey 1999. Salud Pública Méx 2003; 45 Suppl 4:S551-7.

8. Guo S, Cameron W. Tracking of body mass index in children in relation to overweight in adulthood. Am J Clin Nutr 1999; 70 Suppl:145S-8S.

9. Dietz W. Health consequences of obesity in youth: childhood predictors of adult disease. Pediatrics 1998; 101:518-25.

10. Silva NN. Amostragem probabilística: um curso introdutório. 2a Ed. São Paulo: Edusp; 2001.

\section{Agradecimentos}

Este trabalho é parte de Tese de Doutorado apresentada à Escola de Enfermagem de Ribeirão Preto da Universidade de São Paulo, decorrente de convênio firmado com a Faculdade de Enfermagem e Obstetrícia de Celaya, da Universidade de Guanajuato, México.

11. Cole TJ, Bellizi MC, Flegal KM, Dietz WH. Establishing a standard definition for child overweight and obesity worldwide: international survey. BMJ 2000; 320:1240-3.

12. Kaufer M. Un ejercicio de medición antropométrica. Cuad Nutr 1998; 12:12-7.

13. Habicht JP. Estandarización de métodos epidemiológicos cuantitativos sobre el terreno. Bol Oficina Sanit Panam 1974; 76:375-84.

14. Pérez-Lizaur M. Sistema mexicano de alimentos equivalentes. México DF: Fomento de Nutrición y Salud, A.C.; 2003.

15. Secretaria de Salud. PROY-NOM-043-SSS2-1999. Proyecto de norma oficial mexicana. Servicios básicos de salud promoción y educación para la salud en materia alimentaria. Diario Oficial de la Federación 2001; 24 dic.

16. Fornés NS, Martins IS, Velásquez-Meléndez G, Latorre MRO. Escores de consumo alimentar e níveis lipêmicos em população de São Paulo, Brasil. Rev Saúde Pública 2002; 36:12-8.

17. World Health Organization. Physical status: the use and interpretation of anthropometry. Geneva: World Health Organization; 1995 (Technical Report Series, 854).

18. Hosmer DW, Lemeshow S. Applied logistic regression. New York: John Wiley \& Sons; 1989.

19. Concato J, Feinstein AR, Holford TR. The risk of determining risk with multivariate models. Ann Intern Med 1993; 118:201-10.

20. Must A, Dallal GE, Dietz WH. Reference data for obesity, 85th and $95^{\text {th }}$ percentiles of body mass index (wt/ht ${ }^{2}$ ) and triceps skinfold thickness. Am J Clin Nutr 1991; 53:839-46.

21. Kain J, Vio F, Albala C. Obesity trends and determinant factors in Latin America. Cad Saúde Publica 2003; 19 Suppl 1:S77-86.

22. Coleman KJ, Heath EM, Alcalá IS. Overweight and aerobic fitness in children in the United States/ 
Mexico border region. Rev Panam Salud Publica 2004; 15:262-71.

23. Ariza AJ, Chen EH, Binns HJ, Kaufer K. Risk factory for overweight in five-to six-year-old HispanicAmerican children: a pilot study. J Urban Health 2004; 81:150-61.

24. Giammattei J, Blix J, Marshak HH, Wollitzer AO, Pettitt DJ. Television watching and soft drink consumption: association with obesity in 11-to yearold schoolchildren. Arch Pediatr Adolesc Med 2003; 157:882-6.

25. Ribeiro IC, Taddei JA, Colugnatti F. Obesity among children attending elementary public school in São Paulo, Brazil: a case-control study. Public Health Nutr 2003; 6:659-63.

26. Giugliano R, Carneiro EC. Fatores associados à obesidade em escolares. J Pediatr 2004; 80:17-22.

27. O'Loughlin J, Gray-Donald K, Paradis G, Meshefedjian G. One-and two-year predictors of excess weight gain among elementary schoolchildren in multiethnic, low-income, inner-city. Am J Epidemiol 2000; 153:739-46.

28. Wake M, Hesketh K, Waters E. Television, computer use and body mass index in Australian primary school children. J Paediatr Child Health 2003; 39:130-4.

29. Mo-suwan L, Geater AF. Risk factors for childhood obesity in a transitional society in Thailand. Int J Obes Relat Metab Disord 1996; 20:697-703.

30. Stettler N, Signer TM, Suter PM. Electronic games and environment factors associated with childhood obesity in Switzerland. Obes Res 2004; 12:896-903.

31. Ekelund U, Sardinha L, Anderssen S, Harro M, Franks P, Brage S, et al. Associations between objectively assessed physical activity and indicators of body fatness in 9- to 10-y-old European children: a population-based study from 4 distinct regions in Europe (the European Youlth Hearts study). Am J Clin Nutr 2004; 80:584-90.
32. Szklo M, Nieto FJ. Epidemiology: beyond the basics. Gaithersburg: Aspen Publishers; 2000.

33. Dietz WH, Gortmaker SL. Do we fatten our children at the television set? Obesity and television viewing in children and adolescents. Pediatrics 1997; 75:807-12.

34. Gortmaker SL, Must A, Sobol AM, Peterson K, Colditz GA, Dietz WH. Television viewing as a cause of increasing obesity among children in the United States, 1986-1990. Arch Pediatr Adolesc Med 1996; 150:356-62.

35. Bernard L, Lavallée C, Gray-Donald K, Delisle H. Overweight in Cree schoolchildren and adolescents associated with diet, low physical activity and high television viewing. J Am Diet Assoc 1995; 95:800-2.

36. Durant RH, Thompson WO, Johnson M, Baranowski $\mathrm{T}$. The relationship among television watching, physical activity, and body mass composition of 5 or 6 year old children. Pediatric Exercise Science 1996; 8:15-26.

37. Andersen RE, Crespo CJ, Barlett SJ, Cheskin LI, Pratt M. Relationship of physical activity and television watching with body weight and level of fatness among children - results from the Third National Health and Nutrition examination Survey. JAMA 1998; 279:938-42.

38. Hancox RJ, Milne BJ, Poulton R. Association between child and adolescent television viewing and adult health: a longitudinal birth cohort study. Lancet 2004; 364:257-62.

39. Halford JCG, Gulliespie J, Brown V, Pointin E, Dovey T. Effect of television advertisements for foods on food consumption in children. Appetite $2004 ; 42: 221-5$

Recebido em 02/Mar/2005

Versão final reapresentada em 03/Nov/2005 Aprovado em 28/Nov/2005 